Kneebone, I., Hurn, J., Raisbeck, E., Cropley, M., Khoshnaw, H., \& Milton, J. (2010). The validity of goal achievement as an outcome measure in physical rehabilitation day hospitals for older people. International Journal of Disability, Development and Education, 57, 2, 145-153.

\title{
The Validity of Goal Achievement as an Outcome Measure in Physical Rehabilitation Day Hospitals for Older People
}

\author{
Ian I. Kneebone ${ }^{a b}$, Jane S. Hurnc, Elizabeth Raisbeck ${ }^{a}$, Mark Cropley ${ }^{b}$, \\ Hiro Khoshnawa , and Jane E. Milton ${ }^{a}$ \\ aSurrey Community Health, Haslemere, Surrey, UK; bUniversity of Surrey \\ Guildford, UK ${ }^{c}$ Canterbury Christ Church University, Tunbridge Wells, Kent, \\ UK.
}

Physical rehabilitation day hospitals are widely used community-based services designed to meet the medical and rehabilitation needs of older people. While there is evidence for the effectiveness of these services, concerns about the shortcomings of how this is measured has led to the recommendation the achievement of individually tailored goals be used to assess outcomes. This study considered whether such goal achievement demonstrated validity with respect to a standardised measure. The association between goal achievement and change in Nottingham Health Profile-Part 1 (NHP-1) scores was considered for 102 people attending four physical rehabilitation day hospitals. The predicted significant positive relationship between the percentage of goals achieved by participants and NHP-1 scores was not found. This remained evident when functional goals were considered separately to medical goals. Further subsidiary analyses identified a complex relationship between goal achievement and NHP-1 change scores. For those with higher change scores there was a significant negative association between NHP-1 change and goal achievement, while for those with lower (or

\footnotetext{
* Corresponding author. i.kneebone@nhs.net
} 
negative) change scores there was a significant positive association. A curve estimate regression confirmed a highly significant quadratic (curvilinear) relationship. Possible reasons for this finding might include the timing and nature of the goals set, the potential complication of some participants having cognitive impairment, as well as the use of the NHP-1 as a comparison measure. At this time it is recommended goal achievement only be used alongside other measures of day hospital outcome.

Keywords: Day Care; Goals; Validity, Geriatrics, Health Services for the Aged, Treatment Outcome

\section{Introduction}

Physical rehabilitation day hospitals for older people provide community based multi disciplinary assessment, treatment and rehabilitation on a day or part day attendance basis (British Geriatrics Society, 2006). The model of service delivery was developed in the UK in the 1950's and 1960's and has been applied widely including in Australia, Canada, New Zealand, the USA and a number of European countries (Forster, Young, Lambley \& Langhorne, 2008). As is the case with other rehabilitation services day hospitals are required to demonstrate their effectiveness. This is important not only to justify resource allocation, but also in order to ensure patients are aware of the benefits they can expect through a service. Evaluation can also identify areas for improvement as well as areas of success. In this way it contributes to future planning. For instance if patients with goals to reduce their blood pressure improve, but those hoping to improve their mobility do not, attention to physiotherapy needs could be increased in a particular service. Staff morale is also likely supported by a better appreciation of what a service achieves. 
Evidence has accrued demonstrating the benefits of physical rehabilitation day hospitals for older people. They are as equally as effective in delaying death, reducing average hospital stay and reducing institutionalisation as other comprehensive older people services and perform better than when no comprehensive service is provided (Forster et al., 2008). Despite this, dissatisfaction has been expressed with the instruments have been used to evaluate the success of day hospitals for older people, principally because they do not capture the variety of benefits thought to be delivered by the multidisciplinary services or are considered too complex to be used routinely (Black, 2000; Forster, Young \& Langhorne, 1999; Stolee, Rockwood, Fox \& Streiner, 1992). As day hospitals can be considered relatively expensive (Forster et al., 2008) it is important that benefits derived are clear so that decisions about funding are fully informed. One solution to this has been to consider the achievement of individually tailored goals related to rehabilitation in these services to measure success. Goal setting and goal achievement have wide clinical acceptance throughout rehabilitation services (Hurn et al., 2006; Schut, \& Stam, 1994) and have been encouraged in day hospitals for some time (e.g. Martinez, Carpenter \& Williams, 1984). Individual goal setting is thought to be able to capture the unique achievements of individuals within multi-faceted rehabilitation services (Hurn, Kneebone \& Cropley, 2006). While a definitional debate continues (Hurn et al., 2006; Levack et al., 2006), a goal in a rehabilitation context has been considered "a future state that is desired and/or expected” (Wade, 1998, p. 273) and 'goal setting' (used interchangeably with 'goal planning') as the process of agreeing goals between the patient and other interested parties that might include the stipulation of various levels and time-frames (Wade, 1998). While other techniques such as goal attainment scaling (Kiresuk \& Sherman, 1968), allow for partial or over achievement of goals, goal achievement in this context can be determined as the outcome of any intervention with respect to the goals set; goals are achieved or they are not at a given time point. 
Despite its popularity as a form of evaluation among clinicians, questions remain as to whether an idiosyncratic system like goal achievement is as valid in demonstrating outcomes as more standardised measurement instruments (Hurn et al., 2006). While there is some limited evidence that goal achievement demonstrates congruent and concurrent validity with older people in a nursing home setting (Cornbleth, 1978), and concurrent validity in inpatient rehabilitation settings (Wressle, Samuelsson \& Henriksson, 1999), to date its validity in a day hospital context has not received attention. This was the concern of the current investigation. On the basis of clinician observation and the limited literature it was expected that congruent validity of goal achievement, as an outcome measure in day hospitals for older people, would be demonstrated by a significant moderate association between it and an accepted measure of outcome with demonstrated reliability and validity, the Nottingham Health Profile Part 1 (NHP-1)(Alonso et al., 1989). Congruent validity is the establishment of validity (that what is purported to being measured is being measured) by considering a measures correlation with a measure of established validity.

\section{Method}

The study was approved by the West Surrey Local, National Health Service (England), Research Ethics Committee. As the data for the validity trial was that which was routinely collected by the participating services, the Ethics Committee agreed formal consent was not required for its use. Formal written consent however was obtained from participants in the subsidiary reliability check undertaken. 


\section{Participants}

Participants were 102 patients attending one of four day hospitals of a primary care trust in the United Kingdom (UK). Table 1 gives details of the sample. The mean age of the patients was 82 years. The majority of the sample were female (71\%). While the gender ratio is notable it is representative of those who attend these services. The principal reason for referral was musculoskeletal disorder (27\%) followed by falls (23\%). Most (84\%) of the patients lived in their own home or their own flat.

\section{[t] Insert Table 1 about here/[t]}

\section{Primary measures}

Goal Achievement. The goal setting system used followed the SMART (Specific, Measurable, Agreed, Realistic, Timetabled) goals approach under the guidelines proposed by Schut and Stam (1994). At discharge, goal achievement with respect to the goals initially set, was evaluated by the members of the multi-disciplinary team or the nursing staff. All members of the day hospital assessment teams had been trained in the goal setting system. Goal achievement was the percentage of goals attained from 0 to $100 \%$. Medical goals, concerned with change in physiological systems (e.g., 'reduce blood pressure') were differentiated from functional goals, concerned with overt behavioural changes (e.g., 'walk with assistance of a stick') on the basis of consideration by a goal-setting trainer (third author, ER).

Nottingham Health Profile-Part 1 (Alonso et al, 1989). The Nottingham Health Profile (NHP) is a widely-used instrument designed to assess health-related problems in the physical, emotional and social domains. Part 1 of the NHP, known as NHP-1, contains 38 negative statements covering six health dimensions: pain, social isolation, emotional reactions, 
physical mobility, sleep and energy. Items are weighted such that the maximum weighted score for each dimension is 100. This allows a possible total score of 600 .

The NHP-1 is considered appropriate for assessing the impact of medical or social interventions and has been comprehensively evaluated. Studies have established the face, content, criterion and discriminant validity of it as a measure of physical, social and psychological distress associated with medical, social and emotional problems (Alonso et al., 1989). This work has highlighted the suitability of the questionnaire for use with a wide range of individuals including older people (Sharpe, Todd, Caine, \& Tait, 2000) and it has been used for evaluation in day hospital settings with this age group (Palmer \& Kneebone, 2007) Internal consistency achieved in the current sample was acceptable (Time $1, \alpha=.72$, Time 2, $\alpha=.77)$.

\section{Secondary Measures}

Demographic Information Sheet. Information about age, gender, marital status, living circumstances, ethnicity, and major diagnoses, that is collected as standard by the day hospitals was collated in order to allow a description of the population under study.

\section{Procedure}

As was routine for the day hospitals, participants' goals were established with the multidisciplinary team (medical officer, physiotherapist, occupational therapist and nurse) following their initial assessment. Over the course of the study audits of randomly selected patients' goals took place at each of the four study sites, to support goal setting performance. The NHP-1 (routine for the day hospitals) was administered to patients on admission and discharge. 
Concurrent to the study, but with a separate sample, a reliability check was undertaken to establish the consistency of the goal setting between the multidisciplinary teams. Within a week of each other, different day hospital teams assessed eight patients separately. The goals that were established with each patient were then rated independently for their percentage overlap by two therapists affiliated with universities who were familiar with the day hospital environments, but were not currently working within them.

\section{Statistical Analyses and Power}

At a power level of .80 , running Pearson's $r$ with 7 independent variables, and using a maximum significance level of .05, in order to detect a medium sized effect the study required data from 102 patients (Cohen, 1992).

\section{Results}

The hypothesis that there would be a significant association between the percentage of goals achieved and a patient's pre- to post- change scores on the NHP-1 was tested using Pearson's $r$ correlational analysis. The association between percentage of goals achieved for each patient with change (pre- to post-intervention) on each of the 6 dimensions assessed by the NHP-1 and total NHP-1 scores was also considered. As can be seen in Table 2, correlational analyses failed to find associations in the predicted direction. The only significant finding was a low, negative association between change in the sleep dimension of the NHP-1 and percentage of functional goals achieved. As more goals were achieved sleep appeared to deteriorate.

\section{[t] Insert Table 2 near here/[t]}




\section{Exploratory Analyses}

Subsidiary analyses were performed in the attempt to investigate why the expected findings were not evident. On account of the view that the NHP-1 might only reflect changes in functional rather than medical goals, the association between percentage of goals achieved for each patient with change (pre to post intervention) on each of the 6 dimensions assessed by the NHP-1 and total NHP-1 scores was considered separately for each of these goal types. As can be seen in Table 2, once again the predicted association was not evident.

As change in NHP-1 scores from pre to post treatment was 20.2 points and was only modest though significant ( $\left.t_{100}=2.34, p<.05\right)$ and the range was vast ( -242 to 309 ) it was thought that the findings might be a result of a substantial number of patients being on maintenance or delay of deterioration goals (i.e., where goals might be achieved, but where NHP-1 positive change was unlikely). Accordingly, a further analysis was undertaken on a sub-group of the sample, those who achieved above the mean on NHP-1 change. As can also be seen in Table 2 the opposite of what was expected was found; there were significant negative correlations between percentage of overall and functional goals achieved and NHP-1 change scores. Given this unusual finding a further correlational analysis was performed on those persons scoring below the mean for NHP-1 change. This identified a significant positive correlation between NHP-1 change and functional goal achievement. As these findings suggested a likely curvilinear relationship, a curve estimation regression was performed on the functional goal data. As can be seen in Figure 1 this identified a highly significant quadratic relationship $\left(F_{69}=9.72, p=.001\right)$. These results suggest that for patients achieving less change on the NHP-1, percentage of functional goal achievement was associated with greater benefit, but for those achieving greater change, percentage of functional goal achievement did not matter or, in fact, exerted a negative influence. 


\section{[f] Insert Figure 1 near here/[f]}

\section{Reliability Check}

Inter-rater reliability on the separate sample was acceptable for the reliability trial $\left(r_{s}=.71\right.$, p $<.05)$. A low percentage overlap (20\%) between goals set by different teams for the same patients was identified.

\section{Discussion}

This study investigated whether goal achievement demonstrated congruent validity when employed as an outcome measure within a physical rehabilitation day hospital context using the NHP-1 as the comparison measure. The predicted association with the NHP-1 was not identified. This remained evident even when functional goals were considered separately to medical goals. A surprising finding was that goal achievement was negatively associated with outcomes, as measured by NHP-1 change, for some participants and for others, less or nonachievement of goals were associated with greater benefit as measured by NHP-1 change. Such results are difficult to explain. However, possible reasons for them might include the timing and nature of the goals set, the potential complication of some participants having cognitive impairment as well as the appropriateness of the NHP-1 as a reference measure.

Day hospital patient goals are usually established during the first days of admission to a day hospital. At this early stage patients may not be ready to fully engage in the goal setting process, so their true goals may not be apparent and the most relevant goals may not be set. The importance of such readiness to act has been acknowledged in rehabilitation environments (van den Broek, 2005). In addition, around 28\% of day hospital patients may have a significant cognitive impairment (Wain \& Kneebone, 2005), a further reason why it may be difficult to quickly identify appropriate goals. Should irrelevant/inappropriate goals be 
set and be vigorously pursued by staff (and therefore likely be achieved) this may well have a negative effect on outcome. Conversely, should the attention to needs be more flexible over the course of treatment, regardless of the goals originally set (meaning they may be less likely to be achieved) better outcomes might ensue.

Separate to concerns about the nature of goals initially set may be the imprecision of "all or none” goal setting systems. Unlike more sophisticated approaches such as goal attainment scaling (Kiresuk \& Sherman, 1968), the system used in this study made no provision for recording partial achievement or over achievement of goals. Such precision may have enabled our findings to be more sympathetic to our hypothesis. Support for the validity of goal attainment scaling is much stronger than for the goal achievement system, as considered in a recent systematic review (Hurn et al., 2006).

Despite training and an ongoing audit process the findings for the day hospital teams with respect to the reliability of their goal setting suggest a further reason for our unexpected findings. Goal setting may be so idiosyncratic that it depends not only a particular patient's needs at a given point in time but the interaction between these and a particular day hospital team at a particular point in time.

Findings with respect to the differential benefit of goal setting to subgroups within our sample need to be considered in the context that they have been developed on the basis of subsidiary investigative analysis in which the risk of a Type I error is high and the level of power low.

The findings in this study may also have been affected by the use of the NHP-1 as a comparison measure. In a population where a limited amount of change may be evident, that is where a significant proportion of people may have goals relating to maintenance or of 
delaying deterioration, it may be inappropriate to use the NHP-1 given it does not reflect the achievement of such goals.

The failure to identify goal achievement as valid in the current study suggests that if it is to be used as a measure of outcome in day hospital settings, it should be seen as subsidiary to measures that can demonstrate such properties. While it could be argued, on the basis of this study, that goal achievement should not be used, clinically it is difficult to support such a position. Rehabilitation staff use goal setting because they view it as engaging and motivating for their patients (Schut \& Stam, 1994), it has strong face validity with service users (Holliday, Ballinger, \& Playford, 2007) and, notwithstanding our findings, because it shows potential for improving rehabilitation outcomes (Levack et al., 2006).

Further research might consider the examination of goal setting in relation to other measures that can be used in such settings (Palmer \& Kneebone, 2007), as well as examine the topography of goals achieved through rehabilitation day hospitals. A level of validity is almost certain to be established if, for instance, services were to simply list the types of goals that were being achieved in category groups and indicate for what proportion of patients these are being achieved. Obtaining patient views on goal achievement might also consider whether relevant and appropriate goals are being identified. Regardless, the potential of goal achievement to differentially influence rehabilitation outcomes for subgroups of patients, particularly in light of the fact it might exert a negative influence for some, warrants further study.

\section{Conclusion}

While goal achievement offers one solution to the evaluation of physical rehabilitation day hospitals for older people, it is not clear such a measure has the same level of validity as more 
traditional ones. It is recommended that until there is supportive evidence, goal achievement be used alongside other outcome measures in day hospital settings.

\section{Acknowledgement}

This study received funding from the National Health Service (England), Research and Development Fund, Budget 1. Thanks are due to the following for their support: Monika Busch, Sophie Dewar, Ruth Turk, the staff and patients of the Surrey Community Health Day Hospitals including Norman Day Hospital, Milford Assessment and Rehabilitation Centre, Florence Desmond Day Hospital and Cranleigh Day Hospital. Opinions reflect those of the author(s) and do not necessarily reflect those of the funding agency. The authors had no financial or other conflicts of interest.

\section{References}

Alonso, J., Bucquet, D., Hunt, S., McKenna, S., Niero, M., \& Wilund, I. (1989). European Guide to the Nottingham Health Profile. Montpellier, France: European Group for Quality of Life.

Black, D. A. (2000). The modern geriatric day hospital. Hospital Medicine, 61, 539-543.

British Geriatrics Society. (2006). Compendium. 4.4 Geriatric (Medical) Day Hospitals for Older People. Retrieved from

http://www.bgs.org.uk/Publications/Compendium/compend_4-4.htm

Cohen, J. (1992). A power primer. Psychological Bulletin, 112, 155-159.

Cornbleth, T. (1978). Evaluation of goal attainment in geriatric settings. Journal of the American Geriatrics Society, 26, 404-407.

Forster, A., Young, J., Lambley, R. \& Langhorne, P. (2008). Medical day hospital care for the elderly versus alternative forms of care. Cochrane Database of Scientific Reviews Issue 4 Art. no.: CD001730. DOI: 10.1002/146518.CD1730.pub2 
Forster, A., Young, J., \& Langhorne, P. (1999). Systematic review of day hospital care for elderly people: The Day Hospital Group. British Medical Journal, 318, 837-841.

Holliday, R., Ballinger, C., \& Playford, E. D. (2007). Goal setting in neurological rehabilitation: Patient perspectives. Disability and Rehabilitation, 15, 389-394.

Hurn, J., Kneebone, I., \& Cropley, M. (2006). Goal setting as an outcome measure: A systematic review. Clinical Rehabilitation, 20, 756-772.

Kiresuk, T. J., \& Sherman, R. E. (1968). Goal attainment scaling: A general method for evaluating comprehensive community mental health programs. Community Mental Health, 4, 443-453.

Levack, W. M. M., Taylor, K., Siegert, R. J., Dean, S. G., McPherson, K. M., \& Weatherall, M. (2006). Is goal planning in rehabilitation effective? A systematic review. Clinical Rehabilitation, 20, 739-754.

Martinez, F. M., Carpenter, J., \& Williams, J. (1984). The dynamics of a geriatric day hospital. Age and Ageing, 13, 34-41.

Palmer, S., \& Kneebone, I. (2007). An investigation of day hospital outcome measures. International Journal of Therapy and Rehabilitation, 14, 372-377.

Schut, H. A., \& Stam, H. J. (1994). Goals in rehabilitation teamwork. Disability and Rehabilitation, 16, 223-226.

Sharpe, L. D., Todd, C. J., Caine, N., \& Tait, S. (2000). Measurement properties of the Nottingham Health Profile and Short Form 36 health status measures in a population sample of elderly people. British Journal of Health Psychology, 5, 217-233

Stolee, P., Rockwood, K., Fox, R. A., \& Streiner, D. L. (1992). The use of goal attainment scaling in a geriatric care setting. Journal of the American Geriatrics Society, 40, 574578. 
14 I. I. Kneebone et al.

Van den Broek, M. D. (2005). Why does rehabilitation fail? Journal of Head Trauma Rehabilitation, 20, 464-473.

Wade, D. T. (1998). Evidence relating to goal planning in rehabilitation. Clinical Rehabilitation, 12, 273-275.

Wain, H., \& Kneebone, I. (2005). Prevalence of cognitive impairment in medical rehabilitation settings for older people. PSIGE Newsletter, 91, 18-21.

Wressle, E., Samuelsson, K., \& Henriksson, C. (1999). Responsiveness of the Swedish version of the Canadian Occupational Performance Measure. Scandinavian Journal of Occupational Therapy, 6, 84-89. 
Table 1. Characteristics of the Sample

\begin{tabular}{|c|c|c|c|}
\hline & & $\mathrm{N}$ & $\%$ \\
\hline \multirow[t]{3}{*}{ Gender } & Male & 29 & 28 \\
\hline & Female & 72 & 71 \\
\hline & Missing & 1 & 1 \\
\hline \multirow[t]{4}{*}{ Marital Status } & Single & 7 & 7 \\
\hline & Married & 43 & 42 \\
\hline & Divorced & 5 & 5 \\
\hline & Widowed & 47 & 46 \\
\hline \multirow[t]{4}{*}{ Current Living Arrangements } & Own home & 62 & 61 \\
\hline & Flat & 23 & 23 \\
\hline & Warden assisted & 13 & 13 \\
\hline & Nursing home & 4 & 4 \\
\hline \multirow{5}{*}{ Ethnicity } & White British & 96 & 94 \\
\hline & Any other White & 3 & 3 \\
\hline & White or Black Caribbean & 1 & 1 \\
\hline & Indian & 1 & 1 \\
\hline & Missing & 1 & 1 \\
\hline \multirow[t]{18}{*}{ Principal Reason for Referral } & Musculoskeletal & 27 & 27 \\
\hline & Falls & 23 & 23 \\
\hline & Unknown & 10 & 10 \\
\hline & Cardiac & 8 & 8 \\
\hline & Stroke & 8 & 8 \\
\hline & $\begin{array}{c}\text { Neurological (excluding } \\
\text { Parkinson and stroke) }\end{array}$ & 5 & 5 \\
\hline & Gastrointestinal & 5 & 5 \\
\hline & Endocrine (excluding diabetes) & 3 & 3 \\
\hline & Respiratory & 2 & 2 \\
\hline & Parkinsonism & 2 & 2 \\
\hline & Mental/behavioural disorder & 2 & 2 \\
\hline & Dementia & 2 & 2 \\
\hline & Blood disease & 1 & 1 \\
\hline & Rheumatoid arthritis & 1 & 1 \\
\hline & Osteoarthritis & 1 & 1 \\
\hline & Diabetes & 1 & 1 \\
\hline & Acute confusion & 1 & 1 \\
\hline & & & $S D$ \\
\hline \multirow[t]{2}{*}{ Age (years) } & Range & $58-100$ & \\
\hline & Mean & 83 & 7.74 \\
\hline \multirow[t]{2}{*}{ Length of Treatment (days) } & Range & $1-476$ & \\
\hline & Mean & 110 & 93 \\
\hline \multirow{2}{*}{ NHP-1 Change Score } & Range & $-242-309$ & \\
\hline & Mean & 20.02 & 8.88 \\
\hline \multirow{2}{*}{ Percentage of goals achieved (\%) } & Range & $0-100$ & \\
\hline & Mean & 84.25 & 28.11 \\
\hline
\end{tabular}

Note. NHP-1 = Nottingham Health Profile Part 1, SD = Standard Deviation. 
Table 2: Correlation of Nottingham Health Profile Part 1 (NHP-1) Change Scores with Percentage of Goals Achieved.

\begin{tabular}{|c|c|c|c|c|c|c|c|c|}
\hline & & $\begin{array}{l}\text { NHP-1 } \\
\text { Total }\end{array}$ & $\begin{array}{l}\text { Physical } \\
\text { Mobility }\end{array}$ & Pain & Sleep & Energy & $\begin{array}{l}\text { Social } \\
\text { Isolation }\end{array}$ & $\begin{array}{l}\text { Emotional } \\
\text { Reactions }\end{array}$ \\
\hline \multicolumn{9}{|l|}{ Total Sample } \\
\hline \multirow[t]{3}{*}{$(N=102)$} & All Goals & -.15 & -.15 & -.05 & $-.23 *$ & .07 & -.03 & -.01 \\
\hline & Functional Goals & -.18 & -.21 & -.01 & $-.27 *$ & .14 & .02 & -.03 \\
\hline & Medical Goals & -.05 & -.12 & -.08 & -.11 & -.01 & .03 & .02 \\
\hline \multicolumn{9}{|c|}{ Above Mean Change Score } \\
\hline \multirow[t]{3}{*}{$(n=55)$} & All Goals & $-.39 * *$ & -.25 & -.17 & -.24 & .07 & -.02 & -.11 \\
\hline & Functional Goals & $-.40 * *$ & -.31 & -.17 & $-.33 *$ & .16 & -.08 & -.14 \\
\hline & Medical Goals & $-.33^{* *}$ & $-.31 *$ & -.14 & -.16 & -.07 & -.06 & -.14 \\
\hline \multicolumn{9}{|c|}{ Below Mean Change Score } \\
\hline \multirow[t]{3}{*}{$(n=47)$} & All Goals & $.37 * *$ & .02 & .20 & -.17 & .27 & .17 & .21 \\
\hline & Functional Goals & $.53^{* *}$ & .03 & $.40^{*}$ & -.09 & $.43^{*}$ & .28 & .20 \\
\hline & Medical Goals & .13 & .02 & -.07 & -.09 & .02 & -.02 & .17 \\
\hline
\end{tabular}

Note: $* *=p<.01 ; *=p<.05$. 
Figure 1. Curve estimation regression demonstrating a quadratic relationship between Percentage of Functional Goals Achieved and the Nottingham Health Profile - Part 1 change scores.

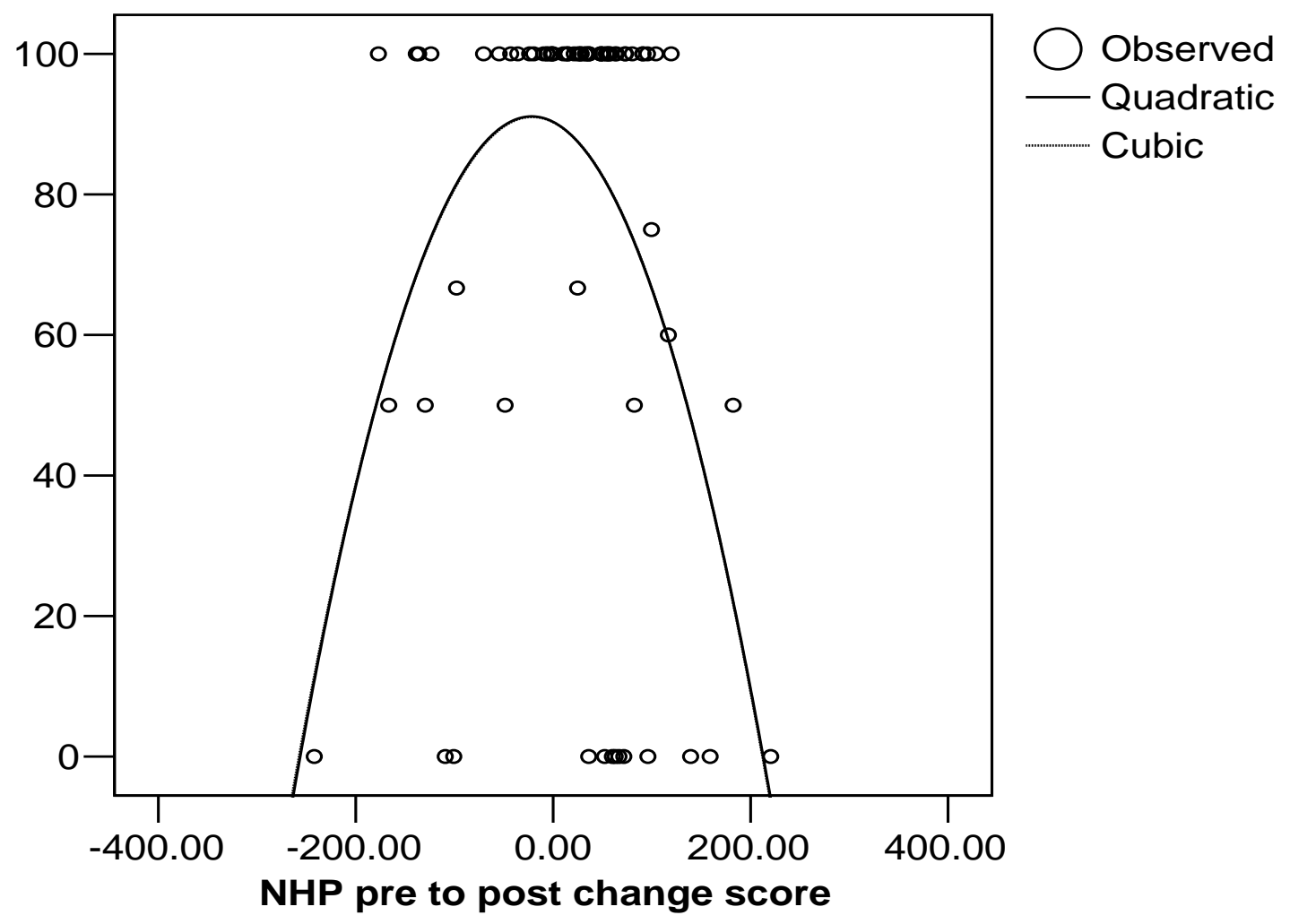

[AU: Please include label for both axis] 